\title{
Don't Want to Get Caught? Don't Say It: The Use of EMOJIS in Online Human Sex Trafficking Ads
}

\author{
Jessica Whitney \\ Affiliation \\ emailaddress@xxx.xxx \\ Aaron Elkins \\ San Diego State University \\ aelkins@mail.sdsu.edu
}

\author{
Murray E. Jennex \\ San Diego State University \\ mjennex@mail.sdsu.edu \\ Eric Frost \\ San Diego State University \\ eric.frost@mail.sdsu.edu
}

\begin{abstract}
Technology has dramatically changed the way criminals conduct their illicit activities. Specifically, the Internet has become a major facilitator of online human sex trafficking. Traffickers are using these technologies to market their victims which presents new challenges for efforts to combat sex trafficking. This study used knowledge management principles and natural language processing methods to develop an improved ontology of online sex trafficking ads. The language of these ads is constantly evolving; therefore, this study explored the role of a new type of indicator, emoticons, to the ontology of human trafficking indicators.
\end{abstract}

\section{Introduction}

The volume of unstructured data and high speed of new data creation makes it difficult for law enforcement to monitor online criminal activities. Also, criminals are constantly changing the language and online platforms they use. With an ever-changing ontology a solid framework for identifying online human trafficking activity is needed. Technology facilitates the real-time collection of data and provides the processing power to quickly solve computational intensive problems.

Additionally, research looks at the importance of certain keywords as a tool for both academics and law enforcement to identify online sex trafficking,. Additionally, these ontologies are ever changing because traffickers are aware of law enforcements use of targeted enforcement technologies and in response they adapt to avoid detection. Due to the highlymonitored nature of online sex trafficking platforms, such as backpage.com and craigslist, as well as the restrictions on what the sites allow to be posted, the vernacular of online sex ads is becoming increasingly subtle. Commonly agreed upon substitute words are constantly being used in ads and keywords are potentially becoming less significant. The language used to advertise victims of human sex trafficking is constantly adapting and identifying them is becoming more about the phraseology, sentiment, and symbols used in the ads.

Unlike previous studies, this research examines the use of emoticons in ads and their significance as a potential indicator of online sex trafficking. This study investigates a knowledge-based framework for the development of a real-time unsupervised learning KMS for identifying victims of online sex trafficking. This type of tool is essential to identifying online trafficking victims in a timely manner and countering the criminals' constant adaptation.

\section{Literature Review}

Between 2010 and 2012 California's regional human trafficking task forces identified nearly 1,300 victims of human trafficking. More than half were found to be victims of sex trafficking and 7 in 10 were U.S citizens recruited domestically [7]. Furthermore, of the 979 incidents of human trafficking reported to the NHTRC [12] [13] in California, 79.8\% were characterized as sex trafficking and only $11 \%$ as labor trafficking. Traffickers use California's major international airports and ports, major interstate and intrastate highways, and state borders to their advantage in moving their victims to cities where they can be exploited for the highest price. This easy access provides criminals to market their victim's services in regions that will provide the highest reward with the lowest risk [6]. The accessibly of transportation routes in California's major metro areas not only provides traffickers with access to a large, highly-profitable client pool, but also acts as tool in keeping their victims disoriented. The easy access to transportation in metro areas makes the frequent movement of victims from city to city very easy for traffickers and 
facilitates their ability to prevent them from building connections and familiarity with a region as this might cause them to feel more comfortable seeking help [6]. Additionally, Roe-Sepowitz, et al. [15] explored demand in the online commercial sex industry by collecting data on responses to decoy online sex ads in 15 different cities. Their finding estimate that 1 of 20 males is soliciting sex online. For San Diego the estimated market size of for online sex trafficking consists of roughly 36,890 men with populations as high as 169,920 johns in some markets [15].

\subsection{Technologies for Combatting Trafficking}

Knowledge management, KM, is defined as the use of knowledge gained through previous decisionmaking experiences for improving organizational effectiveness of current and future decision making activities [11]. Knowledge management systems, KMS, are described by Jennex [11] as systems that promote the successful capture, storage, retrieval, transfer, and reuse of knowledge. A KMS is designed and implemented using a variety of Information Technology (IT)/Information and Communication Technology (ICT) tools. One such tool that supports the design of a KMS is the creation of an ontology [1] [7] [18]. Ontologies are a sub-domain of KM, defined by Noy and McGuinness [14] as a formal explicit description of concepts in a domain of discourse, properties of each concept describing various features and attributes of the concept (slots), and restrictions on the slots. The purpose of an ontology is to build a general representation of domain knowledge to facilitate a common understanding of the domain that can shared across KM applications and groups [16].

There is a growing interest in the use of $\mathrm{KM}$ and data analysis tools for identifying online human trafficking. Studies of online classified advertising data was used to create an ontology of keywords that could be used for identifying sex trafficking ads [9]. The studies compiled lists of indicators of online sex trafficking and highlighted several key terms prevalent in online classified ads that are likely the best indicators of online sex trafficking [9] [10]. Additionally, Dubrawski, et al. [4] used potential cases of human trafficking to determine the weights of keyword terms identified by law enforcement officials to be indicative of trafficking.

Amin [3] discusses the potential benefits of using machine learning as a tool to combat human trafficking. These include; detecting features and unusual patterns indicative of potential cases of human trafficking, identifying rules that can assist in predicting trafficking activity and profiling victims and criminals, and help automate the pattern identification and search process for potential instances of trafficking online. One application of machine learning techniques for combatting online sex trafficking involved the use of a sample of backpage.com ads from March 2016 to train a learning classifier. The ads collected from backpage.com were first filtered using known indicators of online sex trafficking and feature engineering. The six categories used for creating the feature set included; ad language pattern, words and phrases of interest, countries of interest, ad of multiple victims, weight of victim, and whether the ad reference an external website or spa massage therapy. Following this unsupervised filtering, a small portion of the remaining ads were then labeled by human experts as to whether they were instances of human sex trafficking. A semi supervised learning algorithm was then used to label the remaining ads and the classifications were then reviewed by experts in online sex trafficking to evaluate accuracy. Radial basis function (92.41\% True Positive Rate) and K-nearest neighbor (90.42\% True Positive Rate) kernels were used to assign classifications and both learning models were able to predict online instances of sex trafficking with high accuracy [2]. This study indicates the high potential of using machine learning techniques to identify ads that are potentially human trafficking related. Additionally, the use of semi-supervised methods makes the modeling technique more realistic for real world application because obtaining large enough samples of labeled data for a purely supervised learning algorithm is not very plausible.

Dubrawski, et al. [4] trained multiple random forest models using three differed information extraction approaches, term-frequency analysis using law enforcement provided keywords, regular expressions, and natural language processing. Of these three, the natural language processing feature set had the highest accuracy in predicting actual instances of human sex trafficking, lowest false positive rate, followed by the keyword feature extraction method. The natural language processing feature set was obtained by compiling a bag of words from online escort ads and then using principle component analysis for dimensionality reduction [4].

Research on the use of machine learning techniques to combat child sex trafficking has also involved the architectural design and prototyping of Traffic Bot and WAT - Web Archival Tool, its successor. Traffic Bot crawls websites known for ad postings for escort and massages services to collect data and then provides an interface to drill down the collected ad data based on phone numbers as a tool to identify potential cases of sex trafficking. Wang, et al. [17] in designing the Traffic Bot framework examined how classified escort ads disguise phone numbers with the use of unusual 
punctuation and spelling and character or word substitution. They identified a regular expression method to be effective in detecting and decoding these phone numbers and used this technique as their filtering method. They were able to detect ads where the same phone number was listed for different women or multi-person services, two indicators of potential trafficking. They also note that by using the interface to drill down by phone number and identify instances where the area code differed from the location of the ad posting, one may be able to identify the location of a home base [17]. WAT - Web Archival Tool was geared specifically at identifying underage victims of sex trafficking [8]. Hovy et al. [8] clustered data collected via a daily web crawler of websites advertising escort services based on relationships between the postings themselves or poster of the ad. The system facilitated dynamic cluster movement as new data was added and provide three interfaces with which to analyze the resulting clusters [8].

\section{Methodology}

This research uses natural language processing to determine the meaning of emojis used in a sample of online ads. Specific activities are as follows.

The ontology was generated based on a literature review from an initial search conducted using Google Scholar and the university's library's journal article databases with the following search terms in a variety of combinations: "sex trafficking”, "human trafficking”, “California”, “advertising”, “online”, "Internet" and "technology". This was followed by a Boolean search between "human and/or sex trafficking" and the following phrases: "machine learning”, “data science”, “data analytics”, “decision support”, “text mining”, “data mining”, “ontologies”, and "knowledge management". As well as, a search with each of these phrases followed by "for identifying online human sex trafficking”. A review of the above literature identified additional sources.

Also, unstructured interviews were conducted with law enforcement and individuals involved in efforts to combat online human sex trafficking as well as academic researchers with expertise in the area of technology and human trafficking. The law enforcement interviewee was a sergeant from the San Diego County's Sheriff's Department and a member of their joint-agency human trafficking task force. The academic researchers interviewed included two University of Southern California faculty members involved in Department of Justice and Humanity United funded research projects focused on the role of technology in human trafficking activities and the use of data analytics to combat sex trafficking. These interview subjects were selected based on availability of the participant and were conducted by a sole interviewer in order to avoid bias. The participants were asked the following two open ended questions with follow up as needed:

- $\quad$ Explain your main areas of research/methods for targeting and identifying online trafficking activities?

- How have you applied your knowledge of the language used by traffickers (ontologies) to efforts to combat or identify online sex trafficking activities?

Interviews were intended to confirm the practical application of theory from the literature review and identify any knowledge areas the theory did not address. Highlights from the interviews were the identification of the human trafficking task forces' use of emoji indicators for identifying potential instances of online sex trafficking, the need for the consideration of social implications for the use of technology in identifying online human trafficking, difficulties in distinguishing between trafficking and non-trafficking ads and the best approaches for addressing this issue.

Data was collected from the "Women Seeking Men" section of the dating classifieds on Backpage.com. The sample included ads posted between February 2017 and March 2017 for the major cities/counties San Diego, Los Angeles, and Orange County. This sample was selected because of the close proximity of the three cities which makes the language used in the ads subject to less variability due to regional language differences. Ads were scraped from Backpage.com into a csv file using a web scraper. The following attribute data was collected from each of the ads: ad title, location of ad posting, body text of ad, poster's age, date and time of posting, and ad id. The start URLs used to scrape the data were location-based, therefore the data had to be compiled into a single .csv data file. Once compiled, the data file was opened in excel for initial processing. A total of 8,940 postings, roughly a month worth of ads, were retrieved from Backpage.com and exported into a .csv file. This data file was then opened in excel for data cleaning. Data cleaning removed records with missing data. The resulting unstructured final dataset was comprised of 8,744 ad postings and was then imported into $\mathrm{R}$ for final processing and analysis.

After data cleaning, the text from the ad title and the content of the ad were combined into a single column, ad_text, and any capital letters were transformed to lowercase. The stringr package was used in $\mathrm{R}$ to complete the final data pre-processing steps for model development. Based on the literature review and interviews with experts in human 
trafficking, the ads in the dataset were labeled with a value of 1 or 0 to identify whether or not known indicators of online sex trafficking were present. Ads that included the use of at least one keyword / phrase associated with known human sex trafficking indicators were labeled as a 1 and ads containing no known keyword/phrase indicators of trafficking were labeled as a 0 . These resulting values, 1 or 0 , for each ad were assigned to a new column in the dataset. These indicator labels were assigned individually for each sex trafficking indicator category and were based on the presence of the keywords/phrases associated with each specific indicator category. To allow for the processing of emoticons in $\mathrm{R}$, the ad text was converted to ASCII format. This format encodes the emoticons from their native format into their associated utf-8 codes.

Data preparation also included the use of the tm (Text-Mining) package in $\mathrm{R}$ to parse the content of the ads to exclude "punctuation, whitespace, casesensitivity, stop words, and irrelevant terms/characters". It is acknowledged that punctuation, sentence structure, and letter case are meaningful for natural language processing, but these language features are limited from the scope of this research. Stop words are common words that provide little information gain [5], therefore they were removed as a way of eliminating noise from our dataset. The stop words removed included those listed in the English stop word dictionary of the tm package.

The evaluation of emoji indicators also involved the use of the tm package in $\mathrm{R}$ to build a TermDocument matrix with the corpus of ads with each emoji indicator. In order to create a framework for filtering out terms in ads that are not likely to be related to human trafficking, we identified the terms that occurred most frequently across all documents in the corpus to remove from the corpus before analysis. To do this the Term-Document matrix was used to remove sparse terms and then identify the most frequently occurring terms as they are less likely to be specific to human trafficking. These words were selected by identifying not only at the most common terms within the corpus, but also the terms that were present in ads without any indicators of human trafficking. Words common in both these elements were identified as being part of the general ontology for any classified ads related to the provision of sexual services or casual dating and not specific to sex trafficking. For validation of these results, the study looked not only at the most common terms within the corpus, but also the terms that were present in the majority of the documents.

A knowledge management approach was then used to identify emoticons in ads likely to be linked to human sex trafficking. Clusters of ads with and without keywords associated with human sex trafficking indicators were built by applying natural language processing methods to the unstructured dataset. These clusters then determined the frequency of the emoji occurring in each cluster. Ad significance was then determined through logistic regression analysis and $\mathrm{t}$ and $\mathrm{z}$ tests. Findings from this process are discussed in the next section.

\section{Findings}

To compare the ontology to the emojis the subset function in $\mathrm{R}$ was used to create two populations of ads, one with known indicators of trafficking and the second without indicators of trafficking. These known indicators consisted of keywords/phrases in the ontology used. The indicators of human sex trafficking are: sale of services, minor/underage victims, ethnicity/race, country of origin, transient activity/ movement of victims, and non-independent workers/ restricted movement. Table 1 identifies the terms used to subset the dataset for each of these trafficking indicators and Table 2 summarizes the two populations of ads.

Table 1 Ontology Used

\begin{tabular}{|c|c|}
\hline Indicator & Keywords / Phrases \\
\hline Sale of Services & $\begin{array}{l}\text { Donation(s), price, rose(s), dollar(s), } \\
\text { jacks, jacksons, hundreds }\end{array}$ \\
\hline Minor Victims & $\begin{array}{l}\text { Fresh, young, new, tiny, little, new in } \\
\text { town, girl, \& college }\end{array}$ \\
\hline $\begin{array}{c}\text { Ethnicity / Race } \\
\text { African } \\
\text { American } \\
\text { Asian / } \\
\text { Pacific } \\
\text { Islander } \\
\text { Caucasian } \\
\text { Latina }\end{array}$ & $\begin{array}{l}\text { AA, African American, Brown Sugar, } \\
\text { Black (Beauty) } \\
\text { Pocahontas, Asian, Pacific Islander } \\
\text { Caucasian, White, European } \\
\text { Latina, Hispanic }\end{array}$ \\
\hline $\begin{array}{l}\text { Country of Origin } \\
\text { / Nationality }\end{array}$ & $\begin{array}{l}\text { South / East Asia, Eastern / Western } \\
\text { Europe, Central America }\end{array}$ \\
\hline $\begin{array}{l}\text { Transient Activity } \\
\text { / Movement of } \\
\text { Victims }\end{array}$ & $\begin{array}{l}\text { New in town, just arrived, weekend } \\
\text { only, limited time, new arrival, brand } \\
\text { new, in town for the weekend, gone, } \\
\text { back, leaving soon, only for the } \\
\text { weekend, new }\end{array}$ \\
\hline $\begin{array}{l}\text { Non-Independent } \\
\text { Worker/Restricted } \\
\text { Movement }\end{array}$ & $\begin{array}{l}\text { In-call only, no outcall, only in-calls, } \\
\text { come to me, my house }\end{array}$ \\
\hline
\end{tabular}

Table 2. Sample Sizes for Populations of Ads with / without Keyword Indicators

\begin{tabular}{|l|l|l|}
\hline $\begin{array}{l}\text { Human Trafficking } \\
\text { Indicator Category }\end{array}$ & $\begin{array}{l}\text { Population } \\
\text { A (With } \\
\text { Indicators) }\end{array}$ & $\begin{array}{l}\text { Population B } \\
\text { (Without } \\
\text { Indicators) }\end{array}$ \\
\hline
\end{tabular}




\begin{tabular}{|l|l|l|}
\hline Sale of Services & 136 & 8608 \\
\hline Minor Victims & 4215 & 4529 \\
\hline Ethnicity / Race & & \\
\hline African American & 589 & 8155 \\
\hline Asian / Pacific Islander & 886 & 7858 \\
\hline Caucasian & 341 & 8403 \\
\hline Latina / Hispanic & 833 & 7911 \\
\hline Country of Origin & & \\
\hline East / South Asia & 361 & 8383 \\
\hline Eastern / Western Europe & 438 & 8306 \\
\hline Central America & 197 & 8547 \\
\hline $\begin{array}{l}\text { Transient Activity / } \\
\text { Movement of Victims }\end{array}$ & 1108 & 7636 \\
\hline $\begin{array}{l}\text { Non-Independent Worker / } \\
\text { Restricted Movement }\end{array}$ & 62 & 8682 \\
\hline
\end{tabular}

Ontology development identified seven emoticons that were added to the ontology for their link to the sale of services indicator. Table 3 presents the distribution of ads with and without each of these seven emotions for the two populations of ads created based on the presence of sale of services keyword indicators.

Table 3. Summary Statistics of Sale of Services Emoji Indicators

\begin{tabular}{|c|c|c|c|c|}
\hline Emoji & $\begin{array}{l}\text { Emoji } \\
\text { Name }\end{array}$ & $\begin{array}{l}\text { Total } \\
\text { \# Ads } \\
\text { with } \\
\text { Emoji }\end{array}$ & $\begin{array}{l}\text { Population } \\
\text { A: With } \\
\text { Indicators/\% } \\
\text { with Emojis }\end{array}$ & $\begin{array}{l}\text { Population } \\
\text { B: No } \\
\text { Indicators/\% } \\
\text { with Emojis }\end{array}$ \\
\hline$\%$ & rose & 565 & $21 / 15.44 \%$ & $544 / 6.32 \%$ \\
\hline 8 & rosette & 29 & $3 / 2.21 \%$ & $26 / 0.3 \%$ \\
\hline$\overline{\mathbf{s}}$ & $\begin{array}{l}\text { money } \\
\text { bag }\end{array}$ & 48 & $0 / 0 \%$ & $48 / 0.56 \%$ \\
\hline (\$) & $\begin{array}{l}\text { money } \\
\text { with } \\
\text { wings }\end{array}$ & 39 & $1 / 0.74 \%$ & $38 / 0.44 \%$ \\
\hline - & $\begin{array}{l}\text { heavy } \\
\text { dollar } \\
\text { sign } \\
\end{array}$ & 23 & $1 / 0.74 \%$ & $22 / 0.26 \%$ \\
\hline$\$$ & $\begin{array}{l}\text { dollar } \\
\text { banknote }\end{array}$ & 2 & $0 / 0 \%$ & $2 / 0.2 \%$ \\
\hline$\underline{\underline{100}}$ & $\begin{array}{l}\text { hundred } \\
\text { points }\end{array}$ & 1118 & $12 / 8.82 \%$ & $1106 / 12.85 \%$ \\
\hline
\end{tabular}

Since the dollar banknote emoji had a minimal presence in the dataset testing of its significance is a future consideration. Of the remaining six emojis, two were identified in which the proportion of ads with the emoji in the population where the keyword indicators were present was statistically significantly greater than the proportion of ads with the emoji for the population with no keyword indicators of sale of services. These two emoji indicators, the rose $(\chi 2(1)=16.952, \mathrm{p}<$ $0.01)$ and the rosette $(\chi 2(1)=9.4861, \mathrm{p}<0.01)$ emoji, are differing representations of the same keyword indicator of sale of services, roses. Due to the significantly higher presence of the rose emoji in the dataset, this emoji was used to create a logistic regression model (Table 4) for evaluating the relationship between this sale of services emoji and other known indicators of human sex trafficking. The rose emoji was used as the dependent variable in this model and the predictor variables included dummy variables describing the presence of at least one keyword for the following trafficking indicators: sale of services, transient activity and minor victim indicators. Each of these trafficking indicators was found to be a statistically significant predictor of the presence of the rose emoji in an ad as shown in Table 4. The use of at least one sale of services and/or transient activity keyword indicator increases the odds that the rose emoji is used in an ad, while the presence of a minor keyword indicator decreases the odds.

Table 4. Logistic Regression Model Predicting Use of Rose Emoji in an Ad

\begin{tabular}{|l|l|l|l|l|l|}
\hline $\begin{array}{l}\text { Input } \\
\text { Variables }\end{array}$ & Coeff & $\begin{array}{l}\text { Std. } \\
\text { Error }\end{array}$ & $\begin{array}{l}\text { t- } \\
\text { Statistic }\end{array}$ & $\begin{array}{l}\text { P- } \\
\text { Value }\end{array}$ & Odds \\
\hline Intercept & 0.065 & 0.0037 & 17.532 & $\begin{array}{l}< \\
0.001\end{array}$ & 1.07 \\
\hline $\begin{array}{l}\text { Sale_of_Ser } \\
\text { vices (Yes) }\end{array}$ & 0.091 & 0.0212 & 4.301 & $\begin{array}{l}< \\
0.001\end{array}$ & 1.15 \\
\hline $\begin{array}{l}\text { Transient_ } \\
\text { Activity } \\
\text { (Yes) }\end{array}$ & 0.027 & 0.0080 & 3.425 & 0.0006 & 1.03 \\
\hline $\begin{array}{l}\text { Minor_Indi } \\
\text { cator (Yes) }\end{array}$ & -0.011 & 0.0053 & -2.049 & 0.0404 & 0.99 \\
\hline
\end{tabular}

Two-sample testing of proportional differences failed to reject the null hypothesis for the following emoji indicators: the money bag emoji, the money with wings emoji, and the heavy dollar sign emoji. This means that the application of these emoji indicators to the dataset did not provide sufficient evidence to conclude that the proportion of emoticons present in the population with sale of services indicators was greater than the proportion of emoticons in those without. However, a combination of all sale service emoji may yield different results due to the small sample size of ads using emoji indicators. The hundred points emoji was excluded from this additional testing because its use in a significant proportion of ads in the dataset, most of which are in the population with no sale of services indicators, suggests an alternative meaning for this emoji.

The role of three emoticons in identifying minor victims were evaluated. These three emoji indicators were the growing heart emoji, cherry emoji, and cherry blossom emoji. Ads were split into two populations, those with at least one of the following terms; fresh, young, new, tiny, little, new in town, girl, and college; shown through prior research to be indicative of an underage victim and the second with no known minor 
indicators. Two sample z-tests indicated that for all emoji indicators of underage sex trafficking, the observed proportion of each emoji in the population of ads with at least one minor keyword indicator is statistically significantly $(\mathrm{p}<0.01$ ) greater than the observed proportion of the emoji in the population of ads with no minor keyword indicators. Singlepredictor logistic regression modeling was then used to rank the weighted importance of each emoji in the ontology of emoji minor indicators for identifying online human sex trafficking. These single predictor models indicated that the presence of the growing heart $(\beta=0.0813, \mathrm{p}<0.01)$, cherry $(\beta=0.0703, \mathrm{p}<0.01)$, and cherry blossom $(\beta=0.0502, \mathrm{p}<0.05)$ emoji in an ad statistically significantly increases the odds that at least one keyword indicator that the poster of an ad is a minor is used in an ad by $8.47 \%, 7.29 \%$, and $5.15 \%$ respectively. The resulting rankings along with the proportional differences for the presence of each emoji in the two populations are displayed in table 5. A rank of 3 is the highest significance followed by 2 and 1 .

Table 5. Weights and Proportional Test Statistics for Minor Emoji Trafficking Indicators

\begin{tabular}{|l|l|l|l|l|l|}
\hline Emoji & $\begin{array}{l}\text { Emoji } \\
\text { Name }\end{array}$ & $\begin{array}{l}\mathbf{R} \\
\mathbf{a} \\
\mathbf{n}\end{array}$ & $\mathbf{k}$ & $\begin{array}{l}\text { Population } \\
\text { A: With } \\
\text { Keywords/ } \\
\text { \% Ads } \\
\text { with } \\
\text { Emoji (N) }\end{array}$ & $\begin{array}{l}\text { Populatio } \\
\text { n B: } \\
\text { Without } \\
\text { Keywords } \\
\text { /\% Ads } \\
\text { with } \\
\text { Emoji (N) }\end{array}$ \\
\hline $\mathbf{O}$ & $\begin{array}{l}\text { growing } \\
\text { heart }\end{array}$ & 3 & 7.9186 & $\begin{array}{l}4.29 \% \\
(181)\end{array}$ & $\begin{array}{l}3.14 \% \\
(142)\end{array}$ \\
\hline $\mathbf{b}$ & cherry & 2 & 6.6328 & $\begin{array}{l}4.74 \% \\
(200)\end{array}$ & $\begin{array}{l}3.62 \% \\
(164)\end{array}$ \\
\hline & $\begin{array}{l}\text { cherry } \\
\text { blossom }\end{array}$ & 1 & 5.6896 & $\begin{array}{l}7.88 \% \\
(332)\end{array}$ & $\begin{array}{l}6.54 \% \\
(296)\end{array}$ \\
\hline
\end{tabular}

Interviews and background research indicated that the likelihood that the poster of an ad is a minor is much higher if their age is listed as 25 or younger. Similar to the two-sample test conducted between populations of ads with and without keyword indicators of underage sex trafficking, the observed proportion of minor emoji indicator differed for the two populations of ads split based on the poster's age. The proportion of minor emoji indicators was statistically significantly greater in the population of ads where the poster's age was listed as 25 or younger. It is expected that the linguistics in the ads will differ based on the age of the girls as well as the indicators represented.

There were four emojis evaluated for the transient activity/movement of victim indicator: the airplane, small airplane, airplane departure, and airplane arrival. The small airplane and airplane departure had a sample size too small to test. The airplane emoji had a sample size large enough to determine statistical significance. Hypothesis testing for proportional differences in populations indicated that the proportion of ads containing the airplane emoji indicator in the population of ads with at least one keyword indicator of transient activity was significantly greater than the proportion of ads with the emoji in the population of ads with no transient activity keyword indicators $(\chi 2(1)$ $=47.267, \mathrm{p}<0.01)$. Additionally, logistic regression was used to evaluate the relationship between transient activity indicators and the airplane emoji indicator. This modeling technique indicated that the presence of the emoji in an ad is a statistically significant predictor of indicators of transient activity / movement of victims $(\beta=0.375, \mathrm{p}<0.01)$. This means that an ad with the airplane emoji is 1.45 times more likely to contain at least one keyword indicators associated with transient activity/movement of victims. Hypothesis testing indicated no significant difference between the use of the airplane arrival emoji in the two populations of ads for transient activity/movement of victims.

There were six occurrences of the airplane arrival emoji in the dataset but only two had keyword indicators of transient activity. In order to verify the validity of this finding, the ad records in the dataset in which the airplane arrival emoji occurred were compiled into a new data frame. Analysis of the ads in this new data frame found that the airplane emoji was present in one of the ads without any transient activity keyword indicators, but no additional unknown indicators of transient activity. Interestingly, however, all the ads with the airplane arrival emoji had minor keyword indicators. To evaluate the significance of this finding a logistic regression model was built with the airplane arrival emoji as a predictor of the presence of indicators of underage sex trafficking in an ad. This model demonstrated a statistically significant relationship between the airplane arrival emoji and minor that the odds a poster of an ad is underage 1.68 times more likely if the airplane arrival emoji is used $(\beta=0.5183, \mathrm{p}<0.05)$.

A query of ads with keywords associated with restricted movement/non-independent found that a total of 62 ads out of the 8744 records in the dataset included these keyword indicators. This sample size is too small for proportional analysis of these indicators considering the top three most used emoji indicators; the crown, gem stone, and diamond with a dot; all occur in more than 62 ads (but only the crown emoji appears in the sample of 62). Therefore, an alternative approach was used to evaluate the meaning and significance of the crown emoji. Single predictor logistic regression was used to evaluate individually whether or not a significant relationship exists between 
the crown emoji, identified as non-independent worker indicator, and any of the following indicators of human sex trafficking: sale of services, minor victims, and transient activity. This modeling technique used the crown emoji as the independent variable for each of the three indicator categories. The results indicated that a statistically significant relationship exists between the crown emoji and the presence of at least one minor indicator in an ad $(\beta=0.0364, \mathrm{p}<0.05)$. This means that the odds that the poster of an ad is a minor increase by $3.79 \%$ if the crown emoji is used in the ad. Additionally, a t-test was performed to evaluate whether or not there is a significant difference in mean number of indicators present in the ads with and without the crown emoji. This testing indicated that there is a statistically significant difference between the mean number of emoji indicators used in the two populations $(\mathrm{t}(1293.2)=49.119, \mathrm{p}<0.01)$ and $\mathrm{a}$ significant difference between the population means for the total number of indicators, keyword and emoji, used $(\mathrm{t}(1361.3)=30.975, \mathrm{p}<0.05)$.

\section{Discussion}

In 2016, a total of 1,323 human trafficking cases were reported in California to the National Human Trafficking Resource Center. This fits with the estimate made by California's human trafficking task forces that 1,300 cases of trafficking occur each year, roughly $80 \%$ of which are sex trafficking. The dataset used in this study included roughly a month's worth of data from one of the largest known online platforms for sex trafficking ads. The presence of four indicators of human trafficking in an ad as the cutoff for justification of further investigation into the ad using emoji indicators, and keyword indicators individually as well as the combined ontology using five indicators of human trafficking as the cutoff identified an average of 35 ads representing potential cases of online sex trafficking. This would average around 420 potential cases a year; which would account to roughly $32 \%$ of all cases reported in California a year. This is considered a reasonable proportion since the dataset was taken from three major cities considered hotbeds of trafficking in persons for California. Additionally, the presence of four or more trafficking indicators is required for filtering ads using the keyword or emoji ontologies individually and five or more indicators using the combined ontology to justify the consideration of opening an investigation following the implementation of the proposed system due to social implications. While the proposed cutoff value may miss victims, the repercussions of a false positive with this type of KM based system outweigh the risk of false negatives. However, as the system is refined this number can be reconsidered.

The aim of this study is to develop the framework for a KMS that facilitates the automated detection of potential instances of online human sex trafficking. The potential system includes a form of human trafficking indicators not addressed in prior research, emoticons. This representation of trafficking indicators accounts for expected changes in ontologies of the language of online human trafficking ads. Emoticons are intended to combat law enforcement detection efforts and the fact that the application of natural language processing methods to emoticons is much more complex makes it an attractive alternative to keywords. This study evaluated the significance of emoticons in identifying online human sex trafficking activities. These emoticons were identified and categorized from six categories of known human trafficking indicators: sale of services, minor victims, ethnicity/race, country of origin/nationality, transient activity/movement of victims, and restricted movement.

Sale of services and restricted movement emoji indicators were more prevalent in the dataset then their keyword counterparts. The familiarity law enforcement has with keyword indicators of price and restricted movement is likely associated with this finding because it means that traffickers are adapting their language to avoid detection. This supports the findings from this study that emoji indicators are now being used in place of their keyword counterparts and the conclusion that the proposed KM based system provides a better detection mechanism for these indicators of human trafficking. The rose emoji was the most commonly used sale of services indicator and the odds of its use in an ad was statistically significantly related to the presence of keyword indicators for sale of services, transient activity, and minor victim trafficking indicators. While the odds of the use of the rose emoji in an ad increased with the presence of the first two keyword indicators, interestingly, the odds decreased with the use of keywords indicating minor sex trafficking. This finding is likely the result of traffickers using emoji indicators of the ad of minors instead of keywords. The finding that at least one minor indicator was used in nearly half the ads in the dataset, $48.2 \%$, further supports this conclusion. The common use of these words indicates that keywords are less significant in distinguishing between underage sex trafficking and non-trafficking ads. This fits with the marketing strategy of including words associated with youthfulness in ads as a way to attract clientele. The continued inclusion of minor keywords in identifying human trafficking ads may need to be reconsidered. 
One way of potentially filtering through this noise of the use of keywords for advertising purposes versus as an indication of minor victims could be that these keywords are only counted as associated with underage trafficking if the age of the poster of an ad is listed as 25 or younger. Still, the improved ontology of emoji indicators of underage sex trafficking may be the most informative for evaluating whether the subject of an ad is a minor. This means that a potential system may help alleviate current ontologies' inclination towards false positives in identifying minor victims.

\section{Conclusions and Recommendations}

It is concluded that emoji indicators are influential in the development of an improved KMS for the identification of cases on online human sex trafficking. The emoji ontology developed in this study adapts to changes in the language used in online ads for sexual services. This exploratory study highlights a trend towards the use of emoticons as a representation of known trafficking indicators in an online ad. Not only that, but the use of keyword versus emoji indicators of human trafficking activities have shown to be independent of each other. This study's proposed filtering of ads based on the presence of four or more trafficking indicators using the emoji and keyword ontologies independently led to the identification of two distinct groups of ads. Therefore, the use of the proposed ontology allows for the identification of potential victims of trafficking that would not have been found using the previous ontologies. Additionally, the use of a combined ontology of emoticons and keywords associated with known sex trafficking indicators for filtering ads led to the identification of additional potential instances of trafficking. Applying the ontologies and proposed cutoff values for the number of indicators that must be present in an ad for it to warrant further investigation, four using the emoji and keyword ontologies individually and five using the combined ontology, to the dataset in this study narrowed the pool of ads from 8,744 potential cases of trafficking to an average of 35 potential cases. With the limited resources of law enforcement this KM approach provides an effective decision support tool for filtering through the noise of online ads in order to better target anti-trafficking efforts.

Sale of services, underage victim, and restricted movement were shown in this study to be the most significant of the six known human trafficking indicators. This study found that emoticons have become the primary representation of these three indicators in online ads. This is likely due to the familiarity law enforcement has with keywords associated with these three trafficking indicators. Alternatively, this study indicated that keywords are the primary indicator in ads for transient activity /movement of victims, ethnicity/race, and country of origin/nationality. However, the use of emoji indicators for minor, sale of services, and restricted movement indicators does seem to be linked to a higher likelihood of the use of these emoji indicators. Therefore, they are still an important piece to the ontology of emoticons as a filtering mechanism for identifying potential cases of online human sex trafficking. Table 6 summarizes the emoticons found to be used in advertising human sex trafficking victims (does not include emoticons denoting nationality).

Table 6. Summary Emoji Ontology

\begin{tabular}{|c|c|c|}
\hline Emoji & $\begin{array}{l}\text { Emoji } \\
\text { Name }\end{array}$ & Emoji Meaning \\
\hline 4,89 & Rose, rosette & used as subtle indicators of price: \\
\hline O & $\begin{array}{c}\text { Growing } \\
\text { Heart } \\
\end{array}$ & $\begin{array}{l}\text { used by pedophiles to indicate } \\
\text { young girls }\end{array}$ \\
\hline कo, & $\begin{array}{l}\text { Cherry, } \\
\text { Cherry } \\
\text { Blossom }\end{array}$ & $\begin{array}{l}\text { used as a reference to a women's } \\
\text { virginity, and a minor }\end{array}$ \\
\hline,+ 4 & $\begin{array}{l}\text { Airplane, } \\
\text { Airplane } \\
\text { Arrival }\end{array}$ & $\begin{array}{l}\text { Both indicate movement of the } \\
\text { poster with the airplane arrival } \\
\text { tending to show movement of a } \\
\text { minor }\end{array}$ \\
\hline$\theta$ & Crown & $\begin{array}{l}\text { Indicated that the poster is usually } \\
\text { a minor with a pimp controlling } \\
\text { them }\end{array}$ \\
\hline
\end{tabular}

\subsection{Limitations}

Due to limitations of resources and ethical concerns, the data used could not be obtained from actual online human trafficking cases. As a result of this limitation, this study is not intended to evaluate the classification of ads, but to propose an improved KM driven ontology for the identification of potential victims. Second, the proposed ontology is geared towards the identification of female victims of human sex trafficking. While it is recognized that males can also be victims of human sex trafficking, this focus accounts for the majority of victims and the methods used in this study can be applied in the future for the design of an ontology specific to male victims due to its generalizability.

\subsection{Recommendations}

Before the ontology can be implemented it should be further refined through a review of the results by law enforcement in which they would evaluate its 
application based on their prior experiences in identifying online human sex trafficking. Additionally, if experts discern that some of the ads warrant further investigation, the result of those investigations should be used to refine the ontology further. This feedback loop allows for a supervised learning approach through which the ontology performance can be improved and adapt to changes in the environment.

Another approach to using the ontology involves the clustering of ads by applying unsupervised learning methods to the unstructured dataset. A KM approach can then be used to name the clusters to identify those likely to be linked to human sex trafficking. Clusters determined to be more closely related to sex trafficking would be selected by applying the ontology to identify which clusters have a higher number of indicators of human trafficking. Within each of the clusters, ads can then be evaluated against the scoring criteria to determine which ads are likely indicative of human sex trafficking and warrant further investigation. This process acts as a filtering mechanism for dealing with the noise present in the dataset and would direct law enforcement on how to focus their efforts.

One method to further the research involves the creation document-term matrix using term-frequency inverse-document frequency weighting to find terms that are less common across documents. Using this method, higher weights are given to these terms that occur less frequently and the importance of terms that occur in a greater number of documents in the corpus of the ads will be given a lower value [19, p 99]. Terms that occur less frequently across documents and are associated with the ontology of sex trafficking ads derived from the literature review are useful for identifying potential cases of trafficking among the messy data. Therefore, terms given higher weights can be reviewed to identify significant indicators or potential unknown indicators of victims of online sex trafficking. The effectiveness of this is difficult to measure as ads posted by traffickers are likely to have multiple similarities, but it's likely that those words will be less common than the irrelevant content that is present in all ads. Additionally, lower scores may still have meaning since it is impossible to accurately determine how many ads in the dataset involve trafficking related activities. Therefore, a cutoff value may be applicable to creating a decision model on whether to pursue investigation.

Ads vary based on the target audience and author and difficult to group all sex trafficking by most similarity. Additionally, it is difficult to differentiate between certain indicators of sex trafficking and prostitution since there are likely to be multiple commonalities between these two types of ads. Using various clustering techniques, similar ads can be grouped together and the ontology can then be used to identify clusters with frequently occurring terms that are meaningful for identifying online sex trafficking. Two potential methods that can be utilized to build these clusters of ads include Latent Dirichlet Algorithm topic mining and k-means clustering. The lda $\mathrm{R}$ package can be used to cluster the sample of ads by topic using the Latent Dirichlet Algorithm. This would allow one to closely examine the frequent terms in each topic category to try to classify each topic as to whether they are likely to be form of human trafficking or not. The second method of clustering, k-means clustering, facilitates the creation of the optimal number of clusters by looking at the within sum of squares error as a measurement of the similarity of ads within each cluster. Like with the LDA topic clusters, the most frequently occurring terms/symbols within each cluster can be analyzed to assign labels to the kmeans clusters. In addition, by looking at commonly occurring terms within each of those topic cluster additional language used by traffickers that can added to the ontology to improve the filtering of ads can be identified.

Multiple feature sets, such as keywords and natural language processing methods, can be used for clustering ads to identify groups of ads that are likely to be associated with human trafficking. These clusters of ads can then be named and evaluated for those with higher indication of potential cases of trafficking. This evaluation should involve exploration into the presence of emoticons in each cluster to see if a higher number of keywords indicators associated with additional emoji indicators in ads. These clusters could also be weighted using the ontology to decide whether the ads within them warrant an investigation when identified by either filtering or a predictive model. With this information, a decision model could be identified for selecting which clusters warrant investigation and where more attention and resources should be directed for efforts to combat online sex trafficking. With this proposed framework, modeling techniques, such as kNearest Neighbor, can then be used to assign new ads to clusters and provide decision support on how to respond to these ads.

Additional research for the improvement of the ontology should use association analysis to identify any emoticons/symbols that demonstrate a significant relationship with known indicators of trafficking identified in the literature review. This analysis enables the identification of potentially unknown, new indicators of online trafficking that can be added to the ontology and allows for the evaluation of the significance of possible interaction effects between indicators. 
Finally, this research could be generalized to any application that uses an ontology. Examples include identifying the emoticon ontologies in product or service review comments, texts (as a way of understanding cultural representations or as a way of understanding criminal communications), or any other communications or documents that use emoticons.

\section{References}

[1] Almedia, M.B. \& Barbosa, R.R. (2009). Ontologies in knowledge management support: a case study. Journal of the American Society for Information Science and Technology, 60(10), 2032-2047.

[2] Alvari, H., Shakarian, P., \& Snyder, J.E.K. (2016, November). A Non-Parametric Learning Approach to Identify Online Human Trafficking. In IEEE International Conference on Intelligence and Security Informatics: Cybersecurity and Big Data, ISI 2016 (pp. 133-138). Institute of Electrical and Electronics Engineers Inc. Retrieved from https://arxiv.org/pdf/1607.08691.pdf

[3] Amin, S. (2010, January). A Step Towards Modeling and Destabilizing Human Trafficking Networks Using Machine Learning Methods. Paper presented at the AAAI Spring Symposium, Stanford, California. Retrieved from http://www.aaai.org/ocs/index.php/SSS/SSS10/paper/view/1 $\underline{155}$

[4] Dubraski, A., Miller, K., Barnes, M., Boecking, B., \& Kennedy, E. (2015). Leveraging Publicly Available Data to Discern Patterns of Human-Trafficking Activity. Journal of Human Trafficking, 1, 65-85.

[5] Feinerer, I., Hornik, K., Meyer, D. (2008, March). Text Mining Infrastructure in R. Journal of Statistical Software, 25(5), 1-54. Retrieved from https://www.jstatsoft.org/article/view/v025i05

[6] Harris, K. (2012). The State of Human Trafficking in California. Retrieved from State of California Department of Justice Office of the Attorney General website: https://oag.ca.gov/sites/all/files/agweb/pdfs/ht/humantrafficking-2012.pdf

[7] Holsapple, C.W. \& Joshi, K. (2004). A formal knowledge management ontology: Conduct, activities, resources, and influences. Journal of the American Society for Information Science and Technology, 55(7), 593-612.

[8] Hovy, E., Bryan, N.M., Philpot, A., Silva, D.R., \& Sundararajan, A. (2014). Data Integration from Open Internet Sources and Network Detection to Combat Underage Sex Trafficking. In Proceedings of the 15th Annual Internet International Conference on Digital Government Research (pp. 86-90). New York, New York: Association for Computing Machinery.
[9] Hultgren, Marisa. (2015). An Exploratory Study of the Indicators of Trafficking in Online Female Escort Ads (Master's thesis). Available from ProQuest Dissertations and Theses database. (UMI No. 10019611)

[10] Ibanez, M. \& Suthers, D.D. (2014, January). Detection of Domestic Human Trafficking Indicators and Movement Trends Using Content Available on Open Internet Sources. 47th Hawaii International Conference on System Sciences.

[11] Jennex, M.E. (2005). What is KM? International Journal of Knowledge Management, 1(4), i-iv.

[12] National Human Trafficking Resource Center. (2016, February). 2015 NHTRC Annual Report. Retrieved from https://humantraffickinghotline.org/sites/default/files/NHTR C\%202015\%20United\%20States\%20Report\%20-

\%20USA\%20-\%2001.01.15\%20-

\%2012.31.15 OTIP Edited 06-09-16.pdf

[13] National Human Trafficking Resource Center. (2016, April). NHTRC 2015 California State Report. Retrieved from https://humantraffickinghotline.org/sites/default/files/NHTR C\%202015\%20California\%20State\%20Report\%20\%20CA\%20-\%2001.01.15\%20-

\%2012.31.15_OTIP_Edited_06-08-16.pdf

[14] Noy, N.F. \& McGuinness, D.L. (2001) Ontology Development 101: A Guide to Creating Your First Ontology. Retrieved from Stanford University website: http://protege.stanford.edu/publications/ontology_developme nt/ontology101.pdf

[15] Roe-Sepowitz, D., Ryon, S.B., Hickle, K., Gallagher, J.M., \& Hedberg, E.C. (2016). Invisible Offenders: Estimating Online Sex Customers. Journal of Human Trafficking, 2(4), 272-280.

[16] Staab, S., Studer, R., Schnurr, H., \& Sure, Y. (2001) Knowledge Processes and Ontologies. IEEE Intelligent Systems, 16(1), 26-34.

[17] Wang, H., Philpot, A., Hovy, E.H., \& Latonero, M. (2014). Data Mining and Integration to Combat Child Trafficking. Retrieved from Carnegie Mellon University, School of Computer Science website: http://www.cs.cmu.edu/ hovy/papers/12dgo-trafficking.pdf

[18] Wu, J. \& Yang, G. (2005, August). An ontology-based method for project and domain expert matching. In Fuzzy Systems and Knowledge Discovery (pp. 176-185). Retrieved from https://link.springer.com/chapter/10.1007/11540007_22

[19] Zhao, Y. \& Cen, Y. (2013, November). Data Mining Applications with R. Boston: Academic Press. Retrieved from http://site.ebrary.com.libproxy.sdsu.edu/lib/sdsulib/ reader.action?docID=1081554 